\title{
ASSESSING VERBAL FUNCTIONING IN SOUTH AFRICAN SCHOOL BEGINNERS FROM DIVERSE SOCIOECONOMIC BACKGROUNDS: A COMPARISON BETWEEN VERBAL WORKING MEMORY AND VOCABULARY MEASURES
}

\section{Kate Cockcroft}

University of the Witwatersrand

Email: kate.cockcroft@wits.ac.za

\section{Lauren Bloch}

University of the Witwatersrand

\section{Azra Moolla}

University of the Witwatersrand

\section{ABSTRACT}

This study investigated whether measures of verbal working memory are less sensitive to children's socioeconomic background than traditional vocabulary measures. Participants were 120 school beginners, divided into high and low socioeconomic groups. The groups contained equal numbers of English firstlanguage and second-language speakers. All were being educated in English. The results suggest that socioeconomic status accounts for considerable variance in vocabulary measures, while it explains only very small amounts of variance in working memory measures. In addition, the high socioeconomic

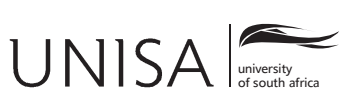

Education as Change www.educationaschange.co.za Volume 20 | Number 1 | 2016 pp. $199-215$

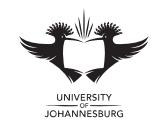

DOI: http://dx.doi.org/10.17159/1947-9417/2016/559 Print ISSN 1682-3206 | Online 1947-9417 (C) 2016 The Authors 
group performed significantly better on the two vocabulary tests relative to the low socioeconomic group, while there were no significant differences between the groups on all but one of the four working memory tests. Working memory assessments appear to be less influenced by environmental factors and may constitute fairer forms of evaluation for children from differing socioeconomic backgrounds. As such, working memory measures may be a valuable supplement to psychoeducational assessment batteries.

Keywords: psychoeducational assessment, socioeconomic status, vocabulary, working memory

\section{INTRODUCTION AND BACKGROUND}

One of the greatest challenges in psychoeducational assessment is the fair testing of children from diverse backgrounds. Unequal opportunities to acquire knowledge will often manifest as lowered performance in such testing situations, which may not reflect the child's actual learning ability. The greatest source of such unequal opportunities arises from differences in socioeconomic status (SES) (Brooks-Gunn and Duncan 1997; Gottfried, Gottfried, Bathurst, Geurin and Parramore 2003). The challenge of how to provide fair assessment opportunities for socioeconomically diverse children is not confined to developing countries, but has become a universal concern as a result of globalisation and the rapid movement of previously disadvantaged individuals to urban, Western environments.

In South Africa, where this study was conducted, there is a stark discrepancy between wealthy and poor, as evidenced by one of the highest Gini coefficients in the world (63.1, where 0 represents perfect equality and 100 implies perfect inequality) (The Human Development Report 2013). South African children from low SES circumstances typically attend government-funded schools infamous for inadequate curricular activities, teachers' unprofessionalism and absenteeism (Fleisch 2008). On the other hand, children from high SES circumstances generally attend 'former model C' schools (which were reserved for white pupils under apartheid) or privately funded schools, and are likely to have access to stimulating home and school environments. The issue of educational systems, which differ vastly in quality (where access to quality is determined by SES), is further complicated in South Africa by language issues. Across all types of educational systems, the majority of South African children are educated in English, although this is the home language of a minority (9.6\%) (Roodt 2011). In addition, the majority of professionals accessed by schoolchildren (speech therapists, occupational therapists, psychologists) speak English only and are reliant on tests that are generally only available in English (Laher and Cockcroft 2013). 
The first year of formal schooling is often the first time that educational difficulties become apparent and the majority of psychoeducational assessments are conducted at this point (Nores and Barnett 2010). These assessments are of critical importance for the child's scholastic career and will determine the necessity for, as well as the type of, intervention that the child should receive. Therefore, it is vital that such assessments are a fair representation of the child's basic learning abilities.

Comprehensive psychoeducational batteries aim to evaluate development and functioning over a broad range of domains, including verbal ability, and thus most such batteries include measures of receptive and/or expressive vocabulary (Flanagan, Ortiz and Alfonso 2013). Vocabulary tests reflect the results of long-term learning and verbal exposure (often referred to as crystallised ability, Gc) in the child's social environment and are closely linked to SES (Dang, Braeken, Ferrer and Liu 2012; Hoff and Tian 2005). Some of the possible reasons for this relationship include the quality and frequency of caregiver conversations with children, caregiver attitudes towards education and the educational value of conversing and reading with children, the availability of material resources, and differences in schooling (Adams, 1990; Bradley and Corwin 2002; Hoff 2003; Noble, Wolmetz, Ochs, Farah and McCandliss 2006). When vocabulary tests are used with school beginners from poorer and non-Western environments whose backgrounds may have limited their exposure to words and concepts, it is often difficult to distinguish typical from atypical language development (Campbell, Dollaghan, Needleman and Janosky 1997). In the search for alternative, fairer ways of evaluating language functioning, the essential proposition of the current study is that verbal working memory tests may be less sensitive to SES influences than traditional vocabulary tests, and may prove to be a useful supplement to such measures.

Working memory is a key cognitive mechanism underlying children's learning; it enables the storage and processing of information, the inhibition of irrelevant information and the performance of sequences of mental actions necessary for the achievement of goals (Baddeley 2000; St Clair Thompson, Stevens, Hunt and Bolder 2010). In addition, working memory enables visual and verbal information to be instantly accessible for problem-solving, and is closely related to fluid cognition (Gf) (that is, processing, rather than knowledge-based cognition) (Horn and Cattell 1982; Hornung, Brunner, Reuter and Martin 2011; Unsworth, Redick, Heitz, Broadway and Engel 2009).

The construct of working memory has been heavily influenced by the work of Baddeley and Hitch (1974), and the model developed subsequently by Baddeley (1986; 2000). According to this model, working memory comprises a set of interrelated mechanisms, of which two are specialised, domain specific short-term stores (verbal and visuo-spatial) and two are domain general mechanisms (namely, the central executive and episodic buffer). The specialised short-term stores are the phonological loop, which briefly retains verbal material, and the visuospatial 
sketchpad, which temporarily stores circumscribed amounts of visual and spatial information. The functioning of the phonological loop and visuospatial sketchpad is overseen by the central executive, which allocates attentional resources to each. The episodic buffer integrates information from the two short-term stores and long-term memory into unified, meaningful episodes (Baddeley 1986; 2000).

Working memory tests typically evaluate the passive, short-term storage aspects of the verbally based phonological loop and/or the visuo-spatial sketchpad (often referred to as 'simple' verbal or visuo-spatial working memory), as well as the active, planning central executive component (frequently referred to as 'complex' verbal or visuo-spatial working memory). Although simple and complex working memories are argued to be theoretically distinct, no single task is an absolutely pure measure of either construct, but would tap simple storage and complex cognitive control to different degrees. Complex tasks primarily reflect cognitive control with fewer storage demands, while simple working memory measures are most reliant on storage and less dependent on cognitive control processes (Unsworth and Engle 2006). In the current study, verbal measures of simple (phonological loop) and complex (central executive) working memory were used.

The focus was on phonological loop tasks as this component of simple working memory has been shown to be related to vocabulary ability and new word learning. The phonological loop comprises a phonological store, which briefly retains information in a phonological code, and a rehearsal process, which refreshes and maintains representations in the phonological store. These components have been shown to be in place in children as young as 4 years (Alloway, Gathercole, Willis and Adams 2004). Drawing on these components, the phonological loop provides immediate storage of new phonological forms of words, which form the basis for learning and storing phonological structures in one's home language and in also additional languages. Thus, the capacity of the phonological loop gives an indication of the child's word learning ability (Baddely, Gathercole and Papagno 1998; Gathercole 2006; Engel de Abreu, Gathercole and Martin 2011).

Individuals with disorders of language learning, such as Specific Language Impairment, typically have both phonological loop and central executive deficits (Archibald and Gathercole 2006; 2007). The central executive plays a more general role in early learning compared with the phonological loop, as it has been shown to support the development of skills involved in reading (Gathercole, Alloway, Willis and Adams 2006; Swanson and Beebe-Frankenberger 2004), numeracy and mathematics (Geary, Hoard, Byrd-Craven and DeSoto 2004; Swanson and Sachse-Lee 2001) and language comprehension (Cain, Oakhill and Bryant 2004). According to Engle (2010), it is the cognitive control mechanisms of the central executive that link working memory to higher order cognitive tasks such as language comprehension and reading. 
Since working memory enables the active maintenance and processing of information, the procedures and stimuli used to test this ability are designed to be equally unfamiliar to all testees and are based on material that is either not explicitly taught, or is very well learned, such as digits and letters. Consequently, such tests are unlikely to bestow significant advantages or disadvantages to children with differing prior knowledge and experience resulting from their SES circumstances. For this reason, working memory assessments are proposed to be far less dependent on environmental factors, such as parental education, quality of education and cognitive stimulation than vocabulary tests, which rely on acquired learning (Engel, Santos and Gathercole 2008; Rinderman, Flores-Mendoza and Mansur-Alves 2010). Thus, it is possible that working memory measures may be relatively pure measures of children's learning capacity and may constitute fairer assessments for children from differing SES backgrounds than tests that are heavily reliant on past learning, such as vocabulary tests.

Empirical findings on the relationship between working memory and SES are equivocal, however. Some researchers have found that working memory performance is uninfluenced by SES (Engel et al. 2008; Messer, Leseman, Boom and Mayo 2010), while others report differential working memory functioning dependant on SES (Noble, McCandliss and Farah 2007; Noble, Norman and Farah 2005). For example, low SES Brazilian children performed comparably to high SES Brazilian children on working memory tests, but significantly poorer on vocabulary measures, when tested in their home language (Engle et al. 2008). Similarly, in Holland, immigrant children from low SES circumstances performed analogously to higher SES, native Dutch speakers on working memory measures, when tested in their home language (Messer et al. 2010).

Possible reasons for the equivocal findings regarding SES and working memory include differences in working memory measures and sample ages. For example, the study by Noble et al. (2007) only utilised simple short-term working memory measures. Such measures do not tap any processing of information, and may have stronger connections with acquired knowledge structures, making them more likely to be influenced by SES background (Alloway, Gathercole and Pickering 2006). In addition, the chronic stress hypothesis proposes that lengthy exposure to poverty can produce chronic stress, which has a negative effect on working memory functioning, which becomes evident later, in adulthood (Evans and Schamberg 2009). This may account for the differences in findings between child and adult samples.

Given these discrepancies in findings concerning the relationship between working memory and SES, as well as the importance of accurate assessments of school beginners' verbal abilities, this study explored these issues in a sample of South African children. It was hypothesised in the current study that the influence of SES on verbal working memory tests would be significantly less than such influence on tests of receptive and expressive vocabulary. 


\section{METHODS}

\section{Participants}

There were 120 Grade 1 children (62 boys; 58 girls; Mean age: 6.73 years, SD: .63). In South Africa, formal schooling starts when a child enters Grade 1 in January of the year in which the child turns 7. Of the participants, 59 came from high SES backgrounds and 61 from low SES circumstances. They were assigned to an SES group based on their results on a SES questionnaire completed by the primary caregiver. All the children in the low SES group attended state-funded schools in a working class area. The high SES children attended state-funded and private schools in an affluent area. Within the high SES group, 29 spoke English at home (EL1), while 30 spoke an African language at home and English was their second language (EL2). Within the low SES group, 37 were EL1 and 24 were EL2. All were being educated at English-medium schools and could communicate easily in English. Children with known learning, emotional, neurological, speech, hearing or motor difficulties were assessed if they wished to participate, but their results were excluded from the study.

\section{Measures}

All the tests described below are standardised measures, but none have been normed on South African children. This matter is discussed under the Procedure section in the context of the ethical use of psychometric tests.

\section{Intellectual ability}

The Ravens Coloured Progressive Matrices (RCPM) (Raven, Court \& Raven 1998) was used to assess nonverbal intelligence in order to determine whether the two groups were equivalent in this regard, and to statistically control for this ability if the groups were not equivalent. For the test, the child completes a geometric figure by choosing a missing piece from six options. There are 36 items in total, in three sets of 12, which increase in difficulty. Each correct response receives a score of one.

\section{Vocabulary tests}

The British Picture Vocabulary Scale (second edition) (BPVS-II) (Dunn, Dunn, Whetton and Burley 1997) is a standardised measure of receptive vocabulary containing line drawings of easily recognisable activities and objects. The child chooses from four pictures that matches a spoken word. There are 60 items and the 
maximum score is the number of objects named correctly, with one score allocated per correct response.

The Boston Naming Test (BNT) (Kaplan, Goodglass and Weintraub 1983) is a standardised test of expressive vocabulary comprising 60 line drawings of common objects, which progress in difficulty. The child names each one. One point is assigned per correct response.

\section{Verbal working memory}

Verbal subtests from the computerised Automated Working Memory Assessment (AWMA) (Alloway, Gathercole and Pickering 2006) were used to measure this ability. The Digit Recall and Nonword Recall subtests assess simple (short-term), verbal working memory. For Digit Recall, the child immediately repeats a sequence of spoken digits in the order presented. There are six trials, each beginning with one digit and increasing to a nine digit sequence. Nonword Recall consists of 40 verbally presented nonwords (words which do not exist in English but which follow English orthographic rules), ranging between 2 to 5 syllables. The child immediately repeats the nonword.

The Counting Recall and Backward Digit Recall subtests evaluate complex (processing) working memory. For Counting Recall, the child counts and remembers the number of circles in a picture comprised of triangles and circles. At the end of each trial, the child recalls the number of circles in each picture, in the correct order. Backward Digit Recall consists of verbally presented sequences of digits. The child immediately repeats the sequence in reverse order.

On each AWMA subtest, a correct response is assigned one point.

\section{Socioeconomic status (SES)}

Socioeconomic status is typically indexed by parental education, occupation and income (Entwisle and Astone 1994; Tomul and Savasci 2012). Due to difficulties asking about income, SES was calculated from three indices: a Living Standards Measure (LSM), the highest occupational status and the highest educational level of the parents/step-parents or guardians, as filled in on a ten minute questionnaire completed by the child's main caregiver. The LSM collected information regarding the number of people living in the house, the number of rooms, the area of residence and the number and type of appliances in the home. This allowed for an LSM score to be calculated. Parental occupation was defined as the highest occupational score of any parent, step-parent or guardian in the home on the following classification: 0 . Unemployed; 1 . Unskilled; 2 . Partly skilled; 3 . Skilled (non-manual and manual); 4. Professional. Parental education was defined as the highest level of education of any parent, step-parent or guardian in the home on the following classification: 1 . No schooling, 2. Less than primary school completed, 3. Primary school completed, 
4. Secondary school not completed, 5. Secondary school completed, and 6. Tertiary education.

The high and low SES groups differed significantly on all SES measures, with caregivers from the high SES group possessing significantly higher levels of education ( $\mathrm{t}=21.59 ; \mathrm{p}=.0001 ; d=3.96)$, professional status $(\mathrm{t}=25.56 ; \mathrm{p}=.0001$; $d=4.65)$ and LSM ( $\mathrm{t}=15.28 ; \mathrm{p}=.0001 ; d=2.79)$. All caregivers in the high SES group had completed secondary school, and half (52\%) had tertiary education and professional occupations. None of the caregivers from the low SES group had tertiary education, most were employed in partly skilled and unskilled jobs (95\%), 5\% were unemployed, few $(6.5 \%)$ had completed secondary education and most had some primary education $(83 \%)$.

\section{Procedure}

Each child was assessed individually, in a quiet area of the school, in a 45-60 minute session. Tests were administered in English and the order was kept constant to ensure a standardised procedure. Data was collected at midyear, within one month, for both groups. Children were assessed by two trained Psychology Masters students, under the supervision of the first author, who is a registered practitioner. The Master's students scored the vocabulary and RCPM measures, which were rescored by the first author in order to establish inter-rater reliability. There was perfect inter-rater agreement between the scoring. The working memory measures (AWMA) were administered and scored by computer, thus ensuring completely uniform scoring across administrations. Consent was obtained from the child's primary caregiver/ guardian and assent from each child.

Ethical issues concerning informed consent and assent, anonymity, confidentiality, opportunities to withdraw and feedback following the Children's Act (No. 38 of 2005) (APA 2002; HPCSA 2011) were appropriately addressed. Some consideration of the fair and ethical use of measures that have not been standardised or normed on South African children (as is the case with all of the measures used in this study) is warranted. The purpose of this investigation was exploratory research, rather than diagnostic. Consequently, participants were not compared with the foreign norms, which would lead to erroneous assumptions regarding performance that is typical or atypical. Instead, groups were compared with one another, allowing for fairer comparisons to be made with children from similar backgrounds. Although approximately half of the participants did not have English as their home language, they were all being educated at English-medium schools, could communicate easily in English and were able to follow the test administration without difficulty. 


\section{RESULTS}

Descriptive statistics for all variables are shown in Table 1. The skewness and kurtosis values for the measures met the criteria for normality, and the assumption of equal variances was met.

Raw test scores are typically used in statistical analyses because they tend to reflect greater variance among individuals as compared with standard scores. On the other hand, standard scores are used in diagnostic situations, in order to meaningfully interpret psychometric test results when comparing individuals with a normative group (Carroll and Carroll 2002). In order to ensure that the use of raw scores in the analyses did not affect the validity of the results, all analyses were conducted twice - once using raw scores and once with standard scores. The results of these analyses did not differ significantly, and so only the analyses conducted with raw scores are reported.

Multivariate analyses of covariances (MANCOVAs) were conducted on the vocabulary and working memory measures in order to compare the SES groups. Since significant differences emerged between the groups on IQ and age (See Table 1), these variables were included as covariates in the MANCOVAs. In order to correct for the effect of multiple tests on the likelihood of a type 1 error, $\mathrm{p}<.02$ was used for the vocabulary measures and $\mathrm{p}<.01$ for the working memory measures, with Bonferroni corrections for two and four tests, respectively. The univariate F tests revealed significantly higher performance by the high SES group on both receptive and expressive vocabulary tests (BPVS and BNT), with large effect sizes (Cohen's d). For the working memory tests, one significant difference emerged between the SES groups. This was on the Nonword Recall subtest, a measure of simple verbal span, and was in favour of the high SES group. The effect size (Cohen's $d$ ) was moderate (Cohen 1992). No significant differences were found between the SES groups on the other test of simple verbal span (Digit Recall) or on the two complex verbal span tests (Counting Recall and Backwards Digit Recall).

Next, the extent to which SES and home language predict performance on the vocabulary and working memory tests was explored. Linear regression analyses were performed on each dependent variable. Model statistics are provided in Table 2.

Together, home language and SES accounted for considerable variance in the vocabulary measures (61\% in BPVS and 57\% in BNT), with SES contributing the majority ( $52 \%$ and $42 \%$, respectively). Home language (EL1 or EL2) only explained $9 \%$ of the variance in receptive vocabulary and $15 \%$ in expressive vocabulary. Socioeconomic status accounted for much smaller amounts of the variance in the processing-dependent working memory tests relative to the storage dependent working memory tests (Processing-dependent: 15\% in Nonword Recall, 13\% in Digit Recall; Storage-dependent: 5\% in Counting Recall, 7\% in Backward Digit Recall). For the working memory tests, home language contributed $4 \%$ of the variance in Backward Digit Recall only. 


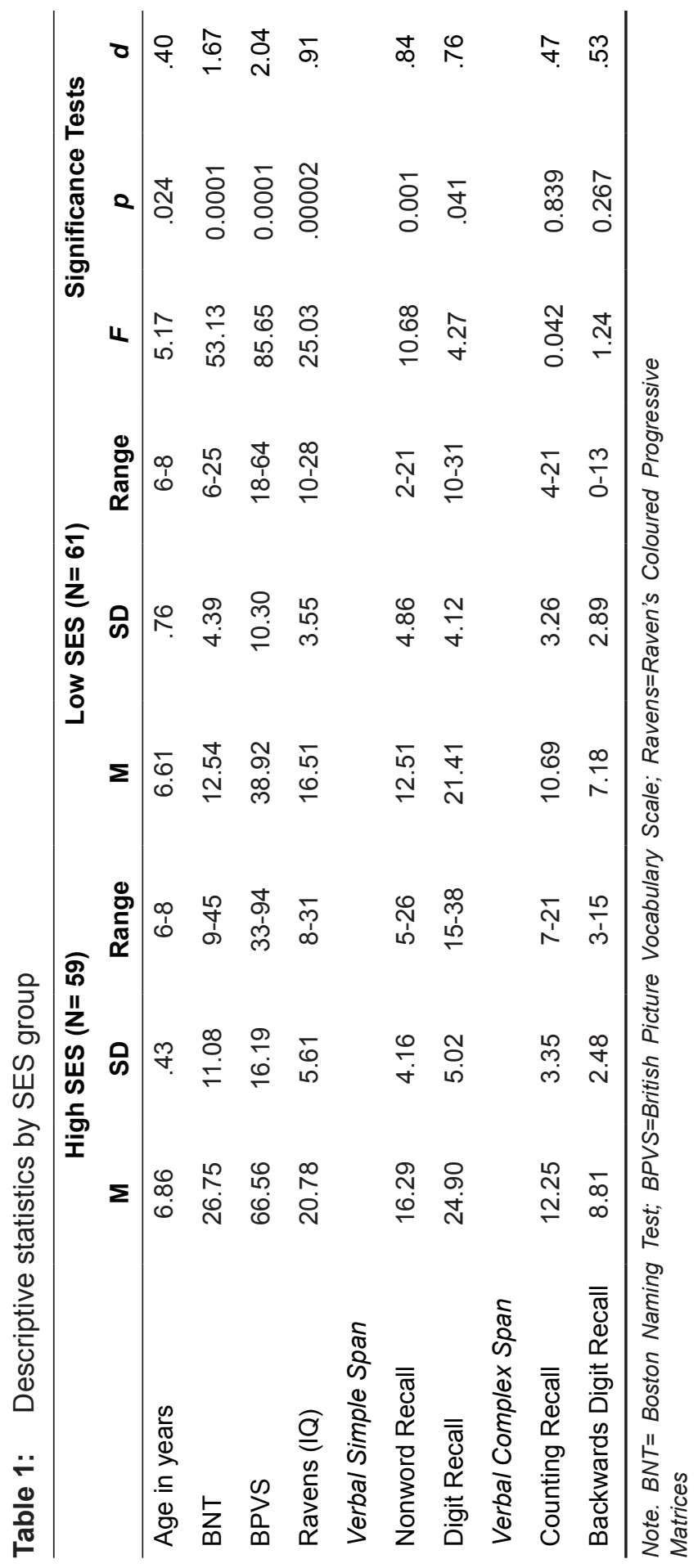




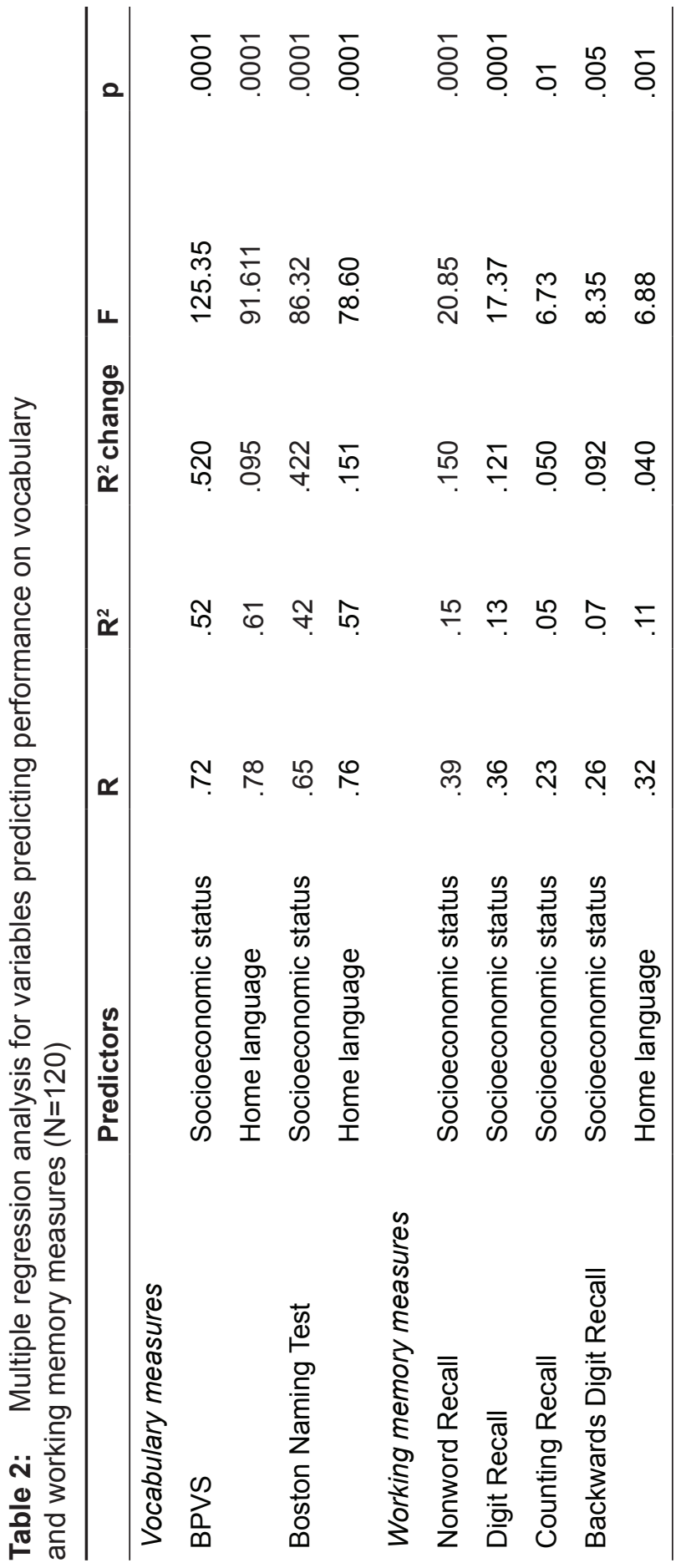




\section{DISCUSSION}

The results suggest that verbal working memory measures are considerably less influenced by SES background than traditional vocabulary tests. This supports previous suggestions that SES-related differences exert a major influence on children's vocabulary ability (Campbell et al. 1997; Engel et al. 2008). Of the working memory tests, those that tap complex verbal working memory involving the central executive appear to be the least influenced by the child's socioeconomic background. This finding confirms emerging evidence that working memory is relatively unaffected by environmental influences related to SES such as attendance at preschool, maternal level of education and income (Engel et al. 2008).

The least SES biased verbal working memory tests appear to be those that draw on complex processing, namely Counting Recall and Backwards Digit Recall. Such working memory tasks require the child to process and temporarily store information, and do not need to draw on long-term knowledge. Such ability seems to be resilient to SES disadvantage. The Digit Recall task, which draws on simple short-term storage, also appeared to be relatively impervious to the effects of SES. A further point worth noting is that all three of these working memory tasks require basic numeracy skills. In this respect, the children from high SES circumstances are likely to have been advantaged as they had all attended preschool, and consequently would have received some instruction in basic numeracy. Conversely, only $46 \%$ of those from low SES backgrounds had attended preschool. Despite this, performance on these three measures was comparable between the high and low SES samples. This finding suggests that performance on these working memory measures may be relatively uninfluenced by socioeconomic disadvantage. It is possible, however, that the young age of the sample may have influenced this result. Consequently, they may not yet have experienced a continuous effect of socioeconomic handicap, unlike the older samples in other studies that reported SES effects on working memory (Evans and Schamberg 2009; Farah et al. 2006).

One of the measures of short-term verbal working memory (Nonword Recall) was slightly affected by SES. This was unexpected as previous studies have found nonword repetition tests to be independent of environmental factors (Campbell et al. 1997; Engel et al. 2008). The anomalous finding may be a result of the inclusion of EL2 children in the high and low SES samples in the current study, as those instances where no SES differences were found in performance on nonword repetition tests, the tests were administered in the child's home language (Archibald and Gathercole 2006; Engel et al. 2008; Gathercole 2006). The finding of SES influences in Nonword Recall also feeds into debates concerning whether or not tests of nonword repetition reflect pure phonological loop capacity (Baddeley et al. 1998) and thus tap phonological storage in a completely knowledge-independent manner (Gathercole 2006), or whether these tests also tap into phonological representations that are stored in long-term memory (Morra and Camba 2009; Messer et al. 2010). 
The results of the current study suggest the latter: those tasks that require long-term memory to support performance would be influenced by acquired knowledge (for which SES is a proxy). Thus, existent vocabulary knowledge stored in long-term memory would play a role in learning new words (or nonwords). Nonword Recall could be performed by drawing analogies with known words, thus accounting for the relatively higher influence of SES and the significantly better performance of the high SES group (which had significantly better vocabulary abilities) on this test. Future research should consider the development of equivalent nonwords in the home languages of the EL2 children, in order for more accurate comparisons to be drawn with previous research.

The results show that vocabulary ability, which is driven by educational opportunity, was clearly affected by SES, while three of the four working memory tests appear not to be affected by such influences. The inclusion and interpretation of such measures in a comprehensive assessment battery together with performance on vocabulary tests may provide a fairer and more realistic picture of a child's verbal learning ability. An added benefit to including measures of working memory in psychoeducational assessments is that they have been shown to be excellent predictors of children's numeracy and literacy abilities (Alloway et al. 2004; Savage, Cornish, Manly and Hollis 2006).

The findings from this study are limited by the relatively small sample sizes in each group, which may have affected statistical power. For example, the effect size on Digit Recall was moderate $(d=.76)$, but did not meet the criterion $(\mathrm{p}<.01)$ for statistical significance. Possibly with a larger sample size, group differences may have emerged on this subtest. The nature of the sample imposed other methodological limitations. Assessments of children at this age need to be brief as they cannot concentrate for the same length of time as adults, and can easily lose motivation if tasks are too difficult or lengthy. For this reason, multiple tests of each construct were not included.

In conclusion, this study makes a contribution to existing research by investigating the effect of SES background on several cognitive skills (verbal working memory, receptive and expressive vocabulary). Socioeconomic status was found to contribute between $42 \%$ and $52 \%$ of the variance in the receptive and expressive vocabulary measures, respectively, supporting suggestions that differences in background and opportunity substantially influence a child's performance on standardised vocabulary tests (Campbell et al. 1997; Hoff and Tian 2005). In addition, SES background accounted for considerably more variance in performance on both the expressive and receptive vocabulary tests than home language. Working memory measures appear far less dependent on SES-related acquired knowledge and skills. Socioeconomic status accounted for between $13 \%$ and $15 \%$ of the variance in the simple, short-term ability tests, and between $5 \%$ and $7 \%$ of the variance in the complex working memory tests. This finding is useful, given attempts to find equitable cognitive assessments 
for children from diverse home and language backgrounds. It is particularly valuable in countries such as South Africa where inequitable social conditions may have impacted on children's cognitive ability, giving unfair advantage to those who have been raised in situations of privilege, and serving to reinforce inequalities. Including measures of verbal working memory processing together with tests of vocabulary in psychoeducational batteries may be more feasible than other proposed solutions to addressing bias in tests, such as developing alternative norms for each of the eleven major language groups in South Africa or relying on subjective observations (Foxcroft 1997). In addition, early scholastic interventions could target the development of verbal working memory strategies as a means of developing language skills (Alloway, Bibile and Lau 2013).

\section{REFERENCES}

Adams, M.J. 1990. Learning to read: Thinking and learning about print. Cambridge, MA.: MIT Press.

Alloway, T.P., Bibile, V. \& Lau, G. 2013. Computerized working memory training: Can it lead to gains in cognitive skills in students? Computers and Human Behavior 29(3): 632-638.

Alloway, T.P., Gathercole, S.E. \& Pickering, S.J. 2006. Verbal and visuospatial short-term and working memory in children: Are they separable? Child Development 77(6): 1698-1716.

Alloway, T. P., Gathercole, S. E., Willis, C. \& Adams, A. 2004. A structural analysis of working memory and related cognitive skills in young children. Journal of Experimental Child Psychology 87(2): 85-106.

APA (American Psychological Association). 2002. Ethical Principles for Psychologists and Code of Conduct. Retrieved from http://www.apa.org/ethics/code2002.html (accessed 16 January 2015).

Archibald, L.M.D. \& Gathercole, S.E. 2006. Nonword repetition: A comparison of tests. Journal of Speech, Language and Hearing Research 49(5): 970-983.

Archibald, L.M.D. \& Gathercole, S.E. 2007. The complexity of complex memory span: Storage and processing deficits in specific language impairment. Journal of Memory and Language 57(2): 177-194.

Baddeley, A.D. 1986. Working memory. Oxford: Oxford University Press.

Baddeley, A.D. 2000. The episodic buffer: A new component of working memory? Trends in Cognitive Sciences 4(11): 417-423.

Baddely, A.D. \& Hitch, G.J. 1974. Working memory. In G.H. Bower (Ed.), The Psychology of Learning and Motivation, vol. 8, 47-90. New York: Academic Press.

Baddeley, A.D., Gathercole, S.E. \& Papagno, C. 1998. The phonological loop as a language learning device. Psychological Review 105(1): 158-173.

Bradley, R.H. \& Corwin, R.F. 2002. Socioeconomic status and child development. Annual Review of Psychology 53(1): 371-399.

Brooks-Gunn, J. \& Duncan, G.J. 1997. The effects of poverty on children. Future Child 7(2): 55-71. 
Cain, K., Oakhill, J. \& Bryant, P. 2004. Children's reading comprehension ability: Concurrent prediction by working memory, verbal ability, and component skills. Journal of Educational Psychology 96(1): 31-42.

Campbell, T., Dollaghan, C., Needleman, H. \& Janosky, J. 1997. Reducing bias in language assessment: Processing dependent measures. Journal of Speech, Language and Hearing Research 40(3): 519-525.

Carroll, S.R. and Carrol, D.J. 2002. Statistics made simple for school leaders. New York: Rowman and Littlefield Education.

Cohen, J. 1992. A power primer. Psychological Bulletin 112(1): 155-159.

Dang, C. Braeken, J., Ferrer, E. \& Liu, C. 2012. Unitary or non-unitary nature of working memory? Evidence from its relation to general fluid and crystallized intelligence. Intelligence 40(5): 499-508.

Dunn, L.M., Dunn, L.M., Whetton, C.W. \& Burley, J. 1997. British Picture Vocabulary Scale (2nd ed.). Windsor, England: NFER-Nelson.

Engle, R.W. 2010. Role of working memory capacity in cognitive control.Current Anthropology 51(S1): S17-S26.

Engel, P.M.J., Santos, F.H. \& Gathercole, S.E. 2008. Are working memory measures free of socioeconomic influence? Journal of Speech, Language and Hearing Research 51(6): 1580 1587.

Engel de Abreu, P.M.J., Gathercole, S.E. \& Martin, R. 2011. Disentangling the relationship between working memory and language: The roles of short-term storage and cognitive control. Learning and Individual Differences 21(5): 569-574.

Entwisle, D. \& Astone, N.M. 1994. Some practical guidelines for measuring youth's race/ethnicity and socioeconomic status. Child Development 65(6): 1521-1540.

Evans, G. \& Schamberg, M. 2009. Childhood poverty, chronic stress, and adult working memory. Proceedings of the National Academy of the United States of America 106(16): 6545-6549.

Farah, M.J., Shera, D.M., Savage, J.H., Betancourt, L. Gianetta, J.M., Brodsky, N.L., Malmud, E.K. \& Hurt, H. 2006. Childhood poverty: Specific associations with neurocognitive development. Brain Research 1110(1): 166-174.

Flanagan, D.P., Ortiz, S.O. \& Alfonso, V.C. 2013. Essentials of cross battery assessment. New York: Wiley.

Fleisch, B. 2008. Primary education in crisis: Why South African children underperform in reading and mathematics. Cape Town: Juta \& Co. Ltd.

Foxcroft, C. 1997. Psychological testing in South Africa: Perspectives regarding ethical and fair practices. European Journal of Psychological Assessment 13(3): 229-235.

Gathercole, S.E. 2006. Nonword repetition and word learning: The nature of the relationship. Applied Psycholinguistics 27(4): 513-543.

Gathercole, S.E., Alloway, T.P., Willis, C. \& Adams, A.M. 2006. Working memory in children with reading disabilities. Journal of Experimental Child Psychology 93(3): 265-281.

Geary, D.C., Hoard, M.K., Byrd-Craven, J. \& DeSoto, M.C. 2004. Strategy choices in simple and complex addition: Contributions of working memory and counting knowledge for children with mathematical disability. Journal of Experimental Child Psychology 88(2): 121-151. 
Gottfried, A.W., Gottfried, A.E., Bathurst, K., Geurin, D.W. \& Parramore, M.M. 2003. Socioeconomic status in children's development and family environment: Infancy through adolescence. In M.H. Bornstein \& R.H. Bradley (Eds.), Socioeconomic Status, Parenting and Child Development, 189-207. Mahwah: Lawrence Erlbaum.

HPCSA (Health Professions Council of South Africa). 2011. Professional Board for Psychology: Rules of Conduct pertaining specifically to Psychology. Retrieved from http://www.hpcsa. co.za/Uploads/editor/UserFiles/downloads/ethical_rules/ethical_rules_of_conduct_2011. pdf (accessed 24 February 2015).

Hoff, E. 2003. The specificity of environmental influence: Socioeconomic status affects early vocabulary development via maternal speech. Child Development 74(5): 1368-1378.

Hoff, E. \& Tian, C. 2005. Socioeconomic status and cultural influences on language. Journal of Communication Disorders 38(4): 271-278.

Horn, J.L. \& Cattell, R.B. 1982. Whimsy and misunderstanding of Gf-Gc theory: A comment on Guilford. Psychological Bulletin 91(3): 623-633.

Hornung, C., Brunner, M., Reuter, R.A.P. \& Martin, R. 2011. Children's working memory: Its structure and relationship to fluid intelligence. Intelligence 39(4): 210-221.

Kaplan, E., Goodglass, H. \& Weintraub, S. 1983. The Boston Naming Test. Baltimore MD: Waverley.

Laher, S. \& Cockcroft, K. 2013. Psychological assessment in South Africa: Research and applications. Johannesburg: Wits University Press.

Messer, M.H., Leseman, P.M.P., Boom, J. \& Mayo, A.Y. 2010. Phonotactic probability effect in nonword recall and its relationship with vocabulary in monolingual and bilingual preschoolers. Journal of Experimental Psychology 105(4): 306-323.

Morra, S. \& Camba, R. 2009. Vocabulary learning in promary school children: Working memory and long-term memory components. Journal of Experimental Child Psychology 104(2): $156-178$.

Noble, K.G., McCandliss, B.D. \& Farah, M.J. 2007. Socioeconomic gradients predict individual differences in neurocognitive abilities. Developmental Science 10(4): 464-480.

Noble, K.G., Norman, M.F. \& Farah, M.J. 2005. Neurocognitive correlates of socioeconomic status in kindergarten children. Developmental Science 8(1): 74-87.

Noble, K.G., Wolmetz, M.E., Ochs, L.G., Farah, M.J. \& McCandliss, B.D. 2006. Brain-behaviour relationships in reading acquisition are modulated by socioeconomic factors. Developmental Science 9(6): 642-654.

Nores, M. \& Barnett, W.S. 2010. Benefits of early childhood interventions across the world: (Under) Investing in the very young. Economics of Education Review 29(2):271-282.

Raven, J.C., Court, J.H. \& Raven, J. 1998. Manual for Raven's Coloured Progressive Matrices and Vocabulary Scales. San Antonio, TX: Harcourt Assessment.

Rinderman, H., Flores-Mendoza, C. \& Mansur-Alves, M. 2010. Reciprocal effects between fluid and crystallised intelligence and their dependence on parents' socioeconomic status and education. Learning and Individual Differences 20(5): 544-548.

Roodt, M. 2011. Research and policy brief: 'Model C' is the model to emulate - $1^{\text {st }}$ February 2011. Retrieved from http://www.sairr.org.za/sairr-today-1/research-and-policy-brief-model-c-isthe-model-to-emulate-1-february-2011 (accessed 20 November 2013). 
Savage, R., Cornish, K., Manly, T. \& Hollis, C.P. 2006. Cognitive processes in children's reading and attention: The role of working memory, divided attention, and response inhibition. British Journal of Psychology 97(3): 365-385.

St Clair Thompson, H., Stevens, R., Hunt, A. \& Bolder, E. 2010. Improving children's working memory and classroom performance. Educational Psychology 30(2): 203-219.

Swanson, H.L. \& Beebe-Frankenberger, M. 2004. The relationship between working memory and mathematical problem solving in children at risk and not at risk for math disabilities. Journal of Educational Psychology 96(3): 471-491.

Swanson, H.L. \& Sachse-Lee, C. 2001. Mathematical problem-solving and working memory in children with learning disabilities: Both executive and phonological processes are important. Journal of Experimental Child Psychology 79(3): 294-321.

The Human Development Report. 2013. United Nations Development Programme. Retrieved from http://hdr.undp.org/en/reports/global/hdr2013/ (accessed 9 December 2013).

Tomul, E. \& Savasci, H.S. 2012. Socioeconomic determinants of academic achievement. Educational Assessment, Evaluation and Accountability 24(3): 175-187.

Unsworth, N. \& Engle, R.W. 2006. Simple and complex memory spans and their relation to fluid abilities: Evidence from list-length effects. Journal of Memory and Language 54(1): 68-80.

Unsworth, N., Redick, T.S., Heitz, R.P., Broadway, J.M. \& Engle, R.W. 2009. Complex working memory span tasks and higher-order cognition: A latent-variable analysis of the relationship between processing and storage. Memory 17(6): 635-654. 